\title{
A novel, efficient and reliable method for thermal process design and optimization. Part II: applications
}

Eva Balsa-Canto ${ }^{\text {a }}$, Julio R. Banga ${ }^{a,}$, Antonio A. Alonso ${ }^{b}$

${ }^{a}$ Chemical Engineering Laboratory, IIM (CSIC), C/Eduardo Cabello 6, 36208 Vigo, Spain

${ }^{\mathrm{b}}$ Department of Chemical Engineering, Universidad de Vigo, Aptdo. 874, 36200 Vigo, Spain

Received 19 January 2001; accepted 27 May 2001

* Corresponding author. Fax: +34-986-292762.

E-mail addresses: eva@iim.csic.es (E. Balsa-Canto),julio@iim.csic.es (J.R. Banga), aalvarez@uvigo.es (A.A. Alonso).

Abstract

In Part I of this study, a method for the derivation of reduced-order models of food thermal processing was presented. In this second part, the capabilities and efficiency of this method is illustrated by applying it to the problems of design and optimization of thermal sterilization. The particular case of conduction-heated foods is considered, without loss of generality. The results clearly indicate that this new methodology allows very fast and accurate solutions of these problems, opening a whole new avenue of possibilities, especially for real-time optimization and control applications. Furthermore, the methodology can be applied to other food processes described by distributed models.

Keywords: Thermal processing; Thermal sterilization; Dynamic optimization; Process design; Proper orthogonal decomposition; Reduced-order models

\section{Introduction}

In the first part of this study, we presented the motivations for this research, and we established, on a general context, the main theoretical ingredients and methods for the derivation of reduced-order models of food thermal processing operations. In this second part, we present the application of those methods to the efficient solution of relevant design and optimization problems. The objective is to give numerical evidence of the great savings in computational time obtained by using these reduced-order models, while guaranteeing the accuracy and generality of the original nominal models. In particular, we will consider the much studied case of thermal sterilization of pre-packaged conduction-heated foods. However, it should be noted that the methodology presented in Part I is general in the sense that it can be applied to any heat 
transfer mechanism (convection, conduction or mixed modes) with domains of arbitrary geometry.

We will focus on two problems of recognized importance: (i) thermal process design, considering constant heating temperature profiles, and (ii) dynamic optimization of thermal processing, i.e. the (open loop) optimal control problem where the time varying sterilization temperature profile is sought in order to maximize a certain performance index while meeting a set of constraints (e.g. microbial lethality).

This paper is structured as follows. We first define, in Section 2, the mathematical model of the system under consideration. For this system, we construct, in Section 3, a reduced-order model which accurately captures the spatial and temporal features of the original distributed system. This reduced model is then employed in the next sections to solve, very efficiently and accurately, the problems of thermal process design and optimization. Finally, we present a discussion of the advantages of this new approach, as well as its implications for the design and optimization of other food processes.

2. Thermal sterilization: model statement

In order to illustrate the applicability and advantages of the techniques presented in Part I to thermal process design and optimization, a case study of industrial relevance was chosen, namely, the thermal sterilization of a conduction-heated canned food in a cylindrical container. This problem can be considered a classic, as it has been previously studied from the design and optimization point of view by a large number of researchers (Durance, 1997; Silva, Oliveira, \& Hendrickx, 1993) and therefore will allow us to establish fair result comparisons. Note, however, that the methodology we propose can be applied to any type of thermal process regardless of the complexity of the heat transfer mechanism, the package geometry or the degree of nonlinearity of the constitutive equations.

In the selected case, the heat transfer in the product can be described as in Eq. (1) of Part I, which now reduces to:

$$
\frac{\partial T}{\partial t}=\frac{K}{\rho C p}\left(\frac{\partial^{2} T}{\partial r^{2}}+\frac{1}{r} \frac{\partial T}{\partial r}+\frac{1}{r^{2}} \frac{\partial^{2} T}{\partial \theta^{2}}+\frac{\partial^{2} T}{\partial z^{2}}\right)
$$

where, for convenience, cylindrical coordinates have been used. Food properties such as density, specific heat and thermal conductivity are assumed to be independent of position and temperature, which in addition to symmetry and isotropy considerations leads to the following partial differential equation:

$$
\frac{\partial T}{\partial t}=\alpha\left(\frac{\partial^{2} T}{\partial r^{2}}+\frac{1}{r} \frac{\partial T}{\partial r}+\frac{\partial^{2} T}{\partial z^{2}}\right)
$$


where $\mathrm{a}$ is the thermal diffusivity. Based on symmetry considerations, boundary conditions can be written for this particular case (cylinder or radius $\mathrm{R}$ and height $2 \mathrm{~L}$ ) as:

$\mathrm{T}(\mathrm{R}, \mathrm{z}, \mathrm{t})=\mathrm{T}_{\text {retort }}(\mathrm{t})$,

$\mathrm{T}(\mathrm{r}, \mathrm{L}, \mathrm{t})=\mathrm{T}_{\text {retort }}(\mathrm{t})$,

$\frac{\partial T}{\partial r}(0, z, t)=0$,

$\frac{\partial T}{\partial z}(r, 0, t)=0$.

The product is assumed to have a uniform initial temperature:

$\mathrm{T}(\mathrm{r}, \mathrm{z}, 0)=\mathrm{T}_{\mathrm{o}}$

The thermal degradation of microbial spores or quality (nutrient or organoleptic) factors are modelled following the well-known TDT equation (Stumbo, 1973):

$\frac{d C_{i}(r, z, t)}{d t}=-\left(\frac{I n 10}{D_{i, r e f}}\right) C_{i}(t) \exp \left(\frac{T(r, z, t)-T_{i, \text { ref }}}{z_{i, \text { ref }}}\right)$

where $\mathrm{C}_{\mathrm{i}}$ indicates the concentration of $\mathrm{i}(\mathrm{i}=\mathrm{M}$ for microorganisms, $\mathrm{N}$ for nutrients, etc.). For nutrients, it is useful to have a final average retention, computed by a suitable volume integral (Schiesser, 1991):

$$
\operatorname{ret}_{N}\left(t_{f}\right)=\frac{1}{V_{T}} \int_{0}^{V_{T}} \exp \left(\frac{-1 n 10}{D_{N}} X \int_{0}^{t_{f}} \exp \left(\frac{T\left(r, z, t-T_{N, \text { ref }}\right.}{Z_{N, \text { ref }}} 1 n 10\right) d t\right) d V
$$

In this work, the degree of microbiological destruction will be evaluated as the lethality at the critical point $\left(F_{c}\right)$, which in this case we can consider to be the geometric center of the cylinder:

$F_{c}\left(t=t_{f}\right)=\int_{0}^{t_{f}} 10 \frac{T_{c}(t)-T_{M, r e f}}{Z_{M, r e f}} d t$

The model parameters for the particular case study considered are presented in Table 1, which correspond to a canned pork puree, as considered by Teixeira, Zinsmeister, and Zahradnik (1975) and Banga, Martin, Gallardo, and Casares (1991), among others.

3. Reduced-order model construction

In this section, we apply the approach presented in Part I of this work to construct a reduced-order model for the canned food system described previously. First, we show how to take advantage of the transformations given in Section 3 of Part I for the boundary conditions and the kinetic equations, respectively, in order to transform the original model in a form suitable for Galërkin projection. Then, for such equivalent system, 
proper orthogonal decomposition (POD) functions are computed and the reduced-order model derived by projecting the set of partial differential equations on the POD subspace. The validation of this reduced model is presented in Section 6.1.

From the considerations given in Section 3.3 of Part I, we transformed the original model (2) with nonhomogeneous first-order boundary conditions (3)-(5) into the equivalent homogeneous PDE:

$$
\frac{\partial \omega}{\partial t}+\frac{\partial T_{\text {retort }}}{\partial t}=L(\omega)=\alpha\left(\frac{\partial^{2} \omega}{\partial r^{2}}+\frac{1}{r} \frac{\partial \omega}{\partial r}+\frac{\partial^{2} \omega}{\partial z^{2}}\right),
$$

where the new variable $\omega$ becomes of the form $\omega=\mathrm{T}-\mathrm{T}_{\text {retort }}$. Note that second-order boundary conditions could be included in the same way through the variable change $\Xi=\mathrm{T}-\chi(\mathrm{t}, \xi)$ with $\chi(\mathrm{t}, \xi)$ being a function constructed to comply with the original, non-homogeneous, boundary. In this equivalent formulation, the control (retort temperature) explicitly appears as its derivative in the resulting PDE. Thermal degradation kinetics can be included into this formulation by inducing a transformation of the form (see Section 3.3 in Part I):

$$
\Theta=\exp \left(\frac{1 n 10}{D_{i, \text { ref }}} w\right)-1
$$

which in combination with (11) leads to the partial differential equation:

$$
\frac{\partial \Theta}{\partial t}+\frac{1 n 10}{D_{i, \text { ref }}}(\Theta+1) \frac{\partial T_{\text {retort }}}{\partial t}=\alpha\left(\frac{\partial^{2} \Theta}{\partial r^{2}}+\frac{1}{r} \frac{\partial \Theta}{\partial r}+\frac{\partial^{2} \Theta}{\partial z^{2}}\right)-\alpha \frac{1 n 10}{D_{i, r e f}}\left(\frac{\partial \omega}{\partial r} \frac{\partial \Theta}{\partial r}-\frac{\partial \omega}{\partial z} \frac{\partial \Theta}{\partial z}\right)
$$

with homogeneous boundary conditions. Note that under this transformation, the exponential-type nonlinearity of the original system reduces to a bilinear form in the spatial derivatives of $\omega$ and $\Theta$ thus simplifying the application of the Galërkin method. In addition, the initial conditions for (11) and (13) now become of the form:

$$
\begin{aligned}
& \omega(r, z, 0)=T_{0}-T_{\text {retort }}(0), \\
& \Theta(r, z .0)=\exp \left(\frac{1 n 10}{D_{i, \text { ref }}} \omega(r, z, 0)\right)-1
\end{aligned}
$$

Finally, the evolution of nutrients concentration is described in terms of the transformed variables as:

$$
\frac{\partial \ln C_{N}}{\partial t}=-\left(\frac{1 n 10}{D_{N, \text { ref }}}\right) \exp \left(\frac{T_{\text {retort }}(t)-T_{N, \text { ref }}}{Z_{N, \text { ref }}}\right)(\Theta(r, z, t)+1),
$$

where $\ln C_{N}$ is used instead of $C_{N}$ for convenience.

The reduced-order model is now derived, as discussed in Part I, by projecting Eqs. (11)-(16) on the set of PODs obtained for the relevant variables $\omega, \Theta$ and $\ln C_{N}$. Each of these variables accepts a truncated series expansion of the form: 


$$
\begin{aligned}
& \widetilde{\omega}(r, z, t)=\sum_{i=1}^{N_{\omega}} a_{\omega i}(t) \phi_{\omega i}(r, z), \\
& \widetilde{\Theta}(r, z, t)=\sum_{i=1}^{N_{\Theta}} a_{\Theta i}(t) \phi_{\Theta i}(r, z), \\
& 1 n C_{N}(r, z, t)=\sum_{i=1}^{N_{N}} a_{N} i(t) \phi_{N i}(r, z),
\end{aligned}
$$

where coefficients $\mathrm{a}_{\oplus \mathrm{i}}, \mathrm{a}_{\Theta \mathrm{i}}$ and $\mathrm{a}_{\mathrm{Ni}}$ evolve in time according to the following set of ordinary differential equations:

$$
\begin{aligned}
& \dot{a}_{\omega i}=-c_{\omega i} \frac{\partial T_{\text {retort }}}{\partial t}+\sum_{j=1}^{N \omega} b_{\omega i} j a_{\omega j}, \quad \mathrm{i}=1, \mathrm{~N}_{\omega} \\
& \dot{a}_{\Theta i}=-\frac{1 n 10}{D_{\text {ref }}} \frac{\partial T_{\text {retort }}}{\partial t}\left(a_{\Theta i}+c_{\Theta i}\right)+\sum_{j=1}^{N \Theta} b_{\Theta i j} a_{\Theta j}+\sum_{k=1}^{N \omega} \sum_{j=1}^{N \Theta} b_{i j k} a_{\omega k} a_{\Theta j}, \mathrm{i}, \mathrm{j}=1, \mathrm{~N}_{\Theta}, \mathrm{k}=1, \mathrm{~N}_{\omega}, \\
& \dot{a}_{N i}=-\left(\frac{1 n 10}{D_{N, r e f}}\right) \exp \left(\frac{T_{r e t o r t}(t)-T_{N, r e f}}{Z_{N, r e f}}\right) \times\left(c_{N} i+\sum_{j} b_{N i j} a_{\Theta j}\right), \quad \mathrm{i}=1, \mathrm{~N}_{\mathrm{N}}, \mathrm{j}=1, \mathrm{~N}_{\Theta} .
\end{aligned}
$$

Terms b and c in Eqs. (20)-(22) are obtained from the Galërkin projection as:

$b_{\omega i j}=\int_{V T} \phi_{\omega i}(r, z) L\left(\phi_{\omega j}(r, z)\right) d V, \mathrm{i}=1, \mathrm{~N}_{\omega}, \mathrm{j}=1, \mathrm{~N}_{\omega}$,

$b_{\Theta i j}=\int_{V T} \phi_{\omega i}(r, z) L\left(\phi_{\omega j}(r, z)\right) d V, \mathrm{i}=1, \mathrm{~N}_{\Theta}, \mathrm{j}=1, \mathrm{~N}_{\Theta}$,

with

$$
\begin{aligned}
& L=\alpha\left(\frac{\partial^{2}}{\partial r^{2}}+\frac{1}{r} \frac{\partial}{\partial r}+\frac{\partial^{2}}{\partial \mathrm{z}^{2}}\right), b_{i j \mathrm{k}}=-\alpha \frac{1 n 10}{D_{r e f}} \int_{V T} \phi_{\Theta i}\left(\frac{\partial \phi_{\omega \mathrm{k}}}{\partial r} \frac{\partial \phi_{\Theta j}}{\partial r}-\frac{\partial \varphi_{\omega \mathrm{k}}}{\partial \mathrm{z}} \frac{\partial \phi_{\Theta j}}{\partial \mathrm{z}}\right) d V, \mathrm{i}, \mathrm{j}=1, \mathrm{~N}_{\Theta}, \mathrm{k}=1, \mathrm{~N}_{\omega}, \\
& b_{N i j}=\int_{V T} \phi_{N i} \phi_{\Theta j} d V, \mathrm{i}=1, \mathrm{~N}_{\mathrm{N}}, \mathrm{j}=1, \mathrm{~N}_{\Theta} \\
& c_{\omega i}=\int_{V T} \phi_{\omega i}(r, \mathrm{z}) d V, \mathrm{i}=1, \mathrm{~N}_{\omega} \\
& c_{\Theta i}=\int_{V T} \phi_{\Theta i}(r, z) d V, \mathrm{i}=1, \mathrm{~N}_{\Theta} \\
& c_{N i}=\int_{V T} \phi_{N i}(r, z) d V, \mathrm{i}=1, \mathrm{~N}_{\mathrm{N}}
\end{aligned}
$$

For this case study, the data required to compute the number and form of the PODs $\phi_{\omega}$, $\phi_{\Theta}$ and $\phi_{\mathrm{N}}$, were obtained from direct numerical simulation (DNS) of the original PDE system. The method of lines (Schiesser, 1991) was used to this purpose. Alternatively, data collected through appropriate experiments could have been used. Typical snapshots obtained from DNS experiments are presented in Figs. 1-3. Fig. 4 shows the relative energy captured by the first eigenfunctions for each of the three variables. As it can be seen from the figure, five eigenfunctions for $\omega$ and $\Theta$, and three for $\ln C_{N}$ are enough to capture about the $99.999 \%$ of the total energy thus ensuring an accurate dynamic description (see the Section 6.1 for details). The selected PODs are presented in Figs. 5-7. 
From this discussion, it follows that the resulting reduced-order model consists of 14 ordinary differential equations (ODEs): five ODEs required to model the temperature distribution inside the container, eight ODEs to describe nutrient degradation and a single equation for the calculation of the lethality at the critical point $\left(\mathrm{F}_{\mathrm{c}}\right)$

\section{Thermal process design}

The classical problem of thermal process design involves the calculation of the processing time at given constant heating temperature in order to ensure certain minimum microbial lethality at final time (Stumbo, 1973). Note that final time here implies that there has been cooling period so that at the end of it, the temperature of the hottest point inside the food is below certain value for which the lethality rate is negligible. Microbial lethality is usually measured either as mass average $\left(\mathrm{F}_{\mathrm{S}}\right)$ or as the lethality at the critical point $\left(\mathrm{F}_{\mathrm{c}}\right)$, which for regular geometries, symmetrical boundary conditions and conduction-heated foods can be taken as the geometrical center. However, it should be noted that, for non-homogeneous and/or anisotropic products, the point with the least lethality at the end of the process is not the geometrical center; in fact, it is non-stationary point (Banga, Alonso, Gallardo, \& Martin, 1993). Having said this, and regarding the case study considered here, it should be recognized that, from practical point of view, we can safely assume that the geometrical center of the can is in fact the critical point.

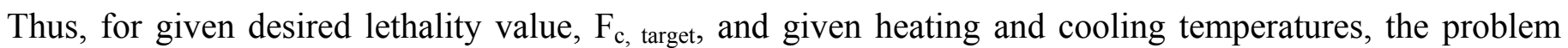
reduces to finding the heating time. The mathematical problem can be stated as follows: find the heating time $\left(t_{h}\right)$ for certain heating temperature ( $\left.T_{\text {heating }}\right)$ to achieve certain target lethality $\left(\mathrm{F}_{\mathrm{c}}\right.$, target $)$ value at final time (i.e. after the cooling period). The problem is equivalent to finding the zeros of $F_{c}=F_{c}$, target as onedimensional function of the heating time:

$0=\mathrm{F}_{\mathrm{c}}\left(\mathrm{t}_{\mathrm{f}}\right)-\mathrm{F}_{\mathrm{c}, \text { target }}=\int_{0}^{t f} 10^{\left(\mathrm{T}_{\mathrm{c}}(\mathrm{t})-\mathrm{T}_{\mathrm{M}, \mathrm{ref}}\right) /\left(\mathrm{Z}_{\mathrm{M}, \mathrm{c}} \mathrm{f}\right)} \mathrm{dt}-\mathrm{F}_{\mathrm{c}, \text { target }}$,

where the temperature at the critical point $\left(T_{c}\right)$ is computed by solving the heat transfer equation, with a boundary condition described by $T_{\text {retort }}(t)$. Ideally, $T_{\text {retort }}(t)$ is described by heating period $t_{h}$ at the given constant heating temperature, followed by step change to cooling period at temperature $T_{\text {cooling }}$ and duration sufficient to ensure that all the points inside the container are below threshold temperature (otherwise, the lethality would continue increasing and the problem would be ill defined). The duration of the cooling period is often taken as percentage of the heating time, with typical values of $20 \%$. This ideal description of the retort temperature cannot be met in practice, due to physical constraints: heating the retort requires 
certain time (come-up time), and switching from the heating to the cooling temperature also needs of certain time (comedown time) to be achieved (Alonso, Banga, \& Martin, 1998).

Thus, more realistic description of the retort temperature for the design problem must include both come-up and come-down periods, which are usually adequately represented by ramps, as depicted in Fig. 8. Note that the slopes of these ramps are taken as fixed, as they come from the physical limitations of the heating and cooling facilities. Thus, even for this more realistic situation, the only degree of freedom continues to be the heating time.

The design of thermal processes for given food product packaged in given geometry is usually done for set of different heating temperatures, and range of different desired lethalities for each of those heating temperatures. Further, the final retention of nutrients and/or quality factors is also usually computed for each of the resulting processes, so the influence of the processing conditions on quality can be addressed. These results are usually presented in plots of quality retention versus heating temperature, with a number of curves corresponding each one to a certain desired lethality (see Teixeira, Dixon, Zahradnik, \& Zinsmeister, 1969). These curves are very useful to visualize the impact of a change in processing conditions over quality, and in fact the processes corresponding to maximum quality retention at each lethality level can be easily identified. Note that this is in fact a way of optimal design, i.e. of optimizing the constant heating temperature processes. But this problem is different (in fact, it is the simplest sub-problem) from the more general case of finding the time varying retort temperature which maximizes a certain performance index, which we consider in Section 5.

Regarding the associated computational effort, solving the design problem for a single given heating temperature and a single desired lethality usually only involves a few simulations of the system model, thus it can be done quite rapidly using personal computers. However, the derivation of the curves mentioned above, which is usually the real objective, requires the repetition of this procedure for a significant number of times, so the final computational effort can become important, especially if the heat transfer model must be solved in 3D. Thus, the use of reduced-order models can play an important role in reducing the effort while guaranteeing accuracy.

With respect to the numerical methods and software implementation performed in this work, we have used Müller's method to solve Eq. (30), as implemented in the IMSL routine DZREAL. Note that for each function evaluation, the solution of the system model is needed (i.e. solution of the conduction heat equation plus the microbial lethality). Once the heating time has been found, the quality (e.g. nutritional and/or 
organoleptic) retention is also computed by integrating the corresponding kinetics over time. The process is repeated for the set of desired heating temperatures and lethality values.

5. Dynamic optimization of thermal processing

The objective of the optimization of thermal sterilization is to achieve a trade off between the beneficial and the destructive effects of the heat processing of food, i.e. to find a heating (e.g. retort) temperature profile that ensures a pre-defined final microbial lethality while maximizing some performance index, e.g. the final nutritional quality of the product. When the problem is reduced to finding the best constant retort temperature (CRT) profile, the solution can be easily visualized by the optimal design procedure outlined in the previous section, or more accurately by simply coupling a univariate optimizer with the thermal process design procedure also outlined above.

The problem of finding the best heating temperature as a function of time is much more difficult. Teixeira et al. (1975) were the first to consider the possible advantages of variable retort temperature profiles regarding the maximization of final nutrient retention, and for that purpose these authors used a simplified ad hoc method based on evaluating different profiles of predefined shapes, without making use of optimization methods. Saguy and Karel (1979) were the first to properly formulate the problem as a dynamic optimization (optimal control) problem, presenting a solution procedure based on the maximum principle of Pontryagin, and concluding that optimal VRT profiles only improved nutrient retention marginally. Nadkarni and Hatton (1985) criticized this approach and presented an alternative based on the distributed maximum principle. However, subsequent work suggest that their results are not optimal, probably due to an error in the simulation of the system during the cooling stage.

Banga et al. (1991) presented a method based on a flexible control vector parameterization, which transforms the optimal control problem into a non-linear programming (NLP) problem. This NLP was solved using a stochastic method, since gradient local methods were found not to converge or converge to local solutions if not initialized properly. These authors considered different objective functions, and showed that, for the cases of maximization of surface quality, or minimization of process time, optimal VRT profiles can be very competitive with respect to the classical CRT profiles. These promising results have been subsequently confirmed by several studies (see reviews of Durance, 1997; Silva et al., 1993) and the topic is still receiving attention (Alvarez-Vazquez \& Martinez, 1999; Chalabi, Willigenburg, Straten, 1999; Kleis Sachs, 2000; Terajima Nonaka, 1996). 
The current state-of-the-art regarding optimal control of distributed systems indicates that the so-called direct methods (and especially control vector parameterization, CVP) are the best solution approach (BalsaCanto, Banga, Alonso, Vassiliadis, 2000). However, direct approaches are often too expensive computationally since an NLP must be solved which requires simulation of the distributed model for each function evaluation. Thus, these approaches are clearly not suitable for real-time applications.

Here, we will show that with the reduced-order model approach, accurate optimal control profiles can be computed very rapidly. In particular, we consider the optimal control problem of maximizing the final retention of nutrient, $\operatorname{ret}_{\mathrm{N}}\left(\mathrm{t}_{\mathrm{f}}\right)$, with constraints on the final microbiological lethality, and the final temperature at the critical point.

The mathematical statement of the problem, in its fixed terminal time form, is as follows:

Find $\mathrm{T}_{\text {retort }}(\mathrm{t})$ over $\mathrm{t} \in\left[\mathrm{t}_{0} ; \mathrm{t}_{\mathrm{f}}\right]$ to maximize

$\mathrm{J}=\operatorname{ret}_{\mathrm{N}}\left(\mathrm{t}_{\mathrm{f}}\right)$

subject to

- the system dynamics (differential constraints) as posed in Section 2, Eqs. (2)-(8);

- final time constraint for the maximum allowable temperature inside the food:

$\mathrm{T}_{\mathrm{c}}\left(\mathrm{r}, \mathrm{z} ; \mathrm{t}=\mathrm{t}_{\mathrm{f}}\right) \leq \mathrm{T}_{\text {max }, \mathrm{tf}} \forall r, \mathrm{z} \in V_{T} ;$

- a final time constraint for the lethality at the critical point:

$\mathrm{F}_{\mathrm{c}}\left(\mathrm{t}=\mathrm{t}_{\mathrm{f}}\right) \geq \mathrm{F}_{\mathrm{c} \text {, target }}$

- upper and lower bounds on the control (retort temperature), the maximum reflecting the physical limitations (due to pressure limits) of the retort, and the minimum given by the cooling medium:

$T_{\text {retort }}^{\min } \leq T_{\text {rerort }} \leq T_{\text {retort }}^{\max }$

6. Results and discussion

\subsection{Reduced-order model validation}

In order to check the reduced-order model given in Eqs. (20)-(22), a typical sterilization retort temperature profile was considered. In its simplest case, the temperature profile consists of two phases: a heating period $\left(t_{h}\right)$ and a cooling period $\left(t_{c}\right)$. A more realistic profile, which includes the heating medium come-up and come-down periods (Fig. 8), is considered here for the comparison of the reduced and the full-order nominal (original) model. 
For the solution of the nominal model, the NMOL method (Schiesser, 1991) was used with a very fine spatial discretization level (41 x 41) and a second-order finite differences approximation, in order to ensure highly accurate results. After applying the NMOL, the nominal model resulted in a set of 3363 ODEs: 1681 for the temperature distribution, plus one extra equation for the evaluation of $F_{c}$ and the remaining 1681 odes for the computation of the nutrient distribution.

Both the reduced and the nominal models were solved using a standard initial value problem (IVP) solver which makes use of a backward differentiation formulas suitable for the solution of stiff problems (LSODA, Hindmarsh, 1983). The time evolution of the temperatures predicted by the reduced-order model, for a number of locations inside the container, including the one at the coldest point, are compared with the ones obtained using the full-order model in Fig. 9. Since the microbial lethality at the critical point $\left(\mathrm{F}_{\mathrm{c}}(\mathrm{t})\right)$ and the final average nutrient retention $\left(\operatorname{ret}_{\mathrm{N}}(\mathrm{t})\right)$ are magnitudes of special interest for design and optimization purposes, a comparison of the full-and reduced-order models results is also presented in Figs. 10 and 11 , respectively. Note that for all these variables the agreement between the reduced-order and the nominal model, solved using a very refined grid, is excellent. This is especially remarkable since the size of the nominal model is 240 times that of the reduced model. Not surprisingly, the rate for solving the reduced model is 64 evaluations per second ( $0.016 \mathrm{~s}$ each evaluation), whereas for the nominal model one evaluation requires $1193 \mathrm{~s}$. It is therefore obvious that the derived reduced model obtained above will be an exceptionally good candidate for the rapid and accurate solution of the design and dynamic optimization problems.

\subsection{Thermal process design results}

It should be noted that for the reduced-order model only five ordinary differential equations are required to simulate the temperature, plus one extra equation for the evaluation of $F_{c}$. Once the solution to Eq. (30) is found, the extended reduced-order model consisting of 13 ODEs is integrated once in order to obtain the corresponding nutrient retention. Note that, for the nominal model, a discretization level of only $11 \mathrm{x} 11$ was used, so as to avoid extremely large computational effort, since it provides reasonable accuracy for process design purposes. The resulting set of ODEs after applying the NMOL consist of 121 equations for temperature, plus one extra equation for the evaluation of $F_{c}$, and a total of 242 ODEs for the case of the calculation of the nutrient retention.

The use of the reduced-order model resulted in speedups of almost 20 times regarding simulation, achieving 
a computing rate of 150 model simulations per second on a PC Pentium III/450 MHz, whereas for the fullorder model the rate was of only eight simulations per second. As a consequence, a very impressive improvement in terms of computation time for the overall process design problem was obtained. Heating times were computed for six different target lethalities $\left(\mathrm{F}_{\mathrm{c} \text {, target }}\right)$ values, ranging from 8 to 18 min, and 12 heating temperatures ( $\mathrm{T}_{\text {heating }}$ ) for each lethality value, ranging from $110^{\circ} \mathrm{C}$ to $140^{\circ} \mathrm{C}$. Using the reducedorder model, the 72 processes were designed in only $4 \mathrm{~s}$ whereas $76 \mathrm{~s}$ where necessary when using the nominal model. Excellent agreement was found between the results obtained with both models. These results are presented in Figs. 12 and 13 where the final nutrient retention and the heating times, respectively, are represented versus the retort temperature for the different target lethality values considered.

\subsection{Dynamic optimization results}

The dynamic optimization problem stated above was solved taking the system parameters presented in Table 1, and a target lethality of $F_{c, \text { target }}=876 \mathrm{~s}$, which corresponds to example I of Banga et al. (1991). The latest version of the dynamic optimization solver ICRS/DS (Banga, Alonso, \& Singh, 1997; Banga, Irizarry, \& Seider, 1998) was used. Tests were also made with other CVP solvers based on Sequential Quadratic Programming (SQP) method, of local nature, but they were found to be very sensitive regarding initialization, so the ICRS/DS solver was preferred due to its robustness. In fact, our main objective here was to evaluate, for a given dynamic optimization solver, the speed-up resulting from the use of reducedorder models. However, it should be noted that for real time applications, SQP solvers equipped with a suitable initialization scheme can be faster. All computation times reported here were obtained using a low cost platform, PC Pentium III/450 MHz.

The ICRS/DS solver is a CVP method which proceeds generating, iteratively, control profiles. At each iteration, the model of the system is solved in order to check the constraints and compute the performance index. Thus, these simulations need to provide all the states variables, i.e. temperatures, nutrients distribution and average retention, and microbial lethality. In the case of the nominal model, a discretization level of 11 x 11 was used, resulting in a set of 243 equations, which could be solved at a rate of five evaluations per second (PC Pentium III/450 MHz). The reduced-order model consisted of 14 ODEs, as described in Section 6.1, with an average of 64 evaluations per second of computation time. Thus, for a fixed number of simulations, speed-ups of almost 13 times can be obtained with the reduced-order model.

When the problem was solved using the reduced order model, a final retention of $47.3 \%$, in agreement with the values reported by Banga et al. (1991), was obtained in less than $50 \mathrm{~s}$ of computation time. The optimal 
control profile, and the corresponding temperature at the critical point, nutrient retention and critical lethality are plotted in Figs. 14-16. Using the nominal model, a similar value was obtained after $720 \mathrm{~s}$ computation. Thus, this speed-up of 15 times obtained with the reduced-order model also indicates that it implies less function evaluations for the same final optimization accuracy, which is an additional advantage.

Although these results show that the use of the reduced-order model is competitive enough, an even better evaluation is obtained by comparing their convergence curves. The performance of the two approaches is compared in Fig. 17, where the performance index (nutrient retention) is plotted versus the computation time for optimization runs using each type of model. This plot clearly highlights the superiority of the reduced-order model in terms of efficiency: starting from an initial profile with a retention of less than $32 \%$, an objective value close to $46 \%$ was already obtained in only $0.27 \mathrm{~s}$, almost the same computational effort required by the nominal model to perform just the first function evaluation $(0.20 \mathrm{~s})$. Further, using the reduced model, a near-optimal retention (above 47\%; which would be adequate for any practical purposes) was obtained in $12 \mathrm{~s}$. These results indicate that the reduced-order model can be effectively used not only for standard dynamic optimization, but also for other demanding tasks like real-time optimization or model predictive control, especially considering that these computation times can be further reduced by using a hybrid ICRS/DS-SQP method.

Regarding the practical implementation of these optimal sterilization policies, suitable model-based control schemes have been developed in recent years. For example, in case of any contingency during the operation, a real-time optimizer would easily re-compute the corresponding optimal retort temperature profile to subsequently update the appropriate temperature and pressure set points of the underlying regulatory scheme. For details on robust and efficient regulation schemes, see (Alonso, Banga, \& Martin (1993a), Alonso, Martin, Shuckla, \& Deshpande (1993b) and Alonso, Banga, \& Martin, 1997). Alternatively, by including the mass and energy balance equations associated to the retort (Alonso et al., 1998), the approach we propose could be integrated in a straightforward manner into a nonlinear model predictive control framework.

\section{Conclusions and future work}

We have shown that the use of reduced-order models, obtained via the POD technique as described in part I of this study, allows very fast and accurate solutions of the thermal process design and optimization problems. The possibilities opened by this new approach reach far beyond the scope of this paper. In fact, these reduced-order models have direct applicability to other important issues in the domain of thermal 
processing, like real-time model-predictive control, stochastic simulation, and parameter estimation, among others.

Moreover, the presented procedure for deriving reduced-order models is valid not only for thermal processing (including relevant variants like ohmic heating, microwave heating, etc.), but to the vast majority of food processes, which have a distributed, non-linear nature (e.g. cooling, freezing and thawing, drying, baking, etc.).

\section{Acknowledgements}

This work was supported in part by the EU (project FAIR CT96-1192) and the Spanish Government (CICyT project ALI97-1939-CE). Author Balsa-Canto thanks the Diputacion Provincial de Pontevedra, Spain, for a pre-doctoral fellowship.

\section{References}

Alonso, A. A., Banga, J. R., \& Martin, R. I. P. (1993a). A new strategy for the control of the cooling stage of the sterilization process in steam retorts. Transactions of the IChemE, 71C, 197-205.

Alonso, A. A., Banga, J. R., \& Martin, R. P. (1997). A complete dynamic model for the thermal processing of bioproducts in batch units and its application to controller design. Chemical Engineering Science, 52(8), 1307-1322.

Alonso, A. A., Banga, J. R., \& Martin, R. I. P. (1998). Modeling and adaptive control of a batch sterilization process. Computers and Chemical Engineering, 22(3), 445-458.

Alonso, A. A., Martin, R. I. P., Shuckla, N. V., \& Deshpande, P. B. (1993b). On line quality control of (nonlinear) batch systems: application to thermal processing of canned foods. Journal of Food Engineering, $19,275-289$.

Alvarez-Vazquez, L. J., \& Martinez, A. (1999). Modelling and control of natural convection in canned foods. IMA Journal of Applied Mathematics, 63(3), 247-265.

Balsa-Canto, E., Banga, J. R., Alonso, A. A., \& Vassiliadis, V.S. (2000). Optimal control of distributed processes using restricted second order information. In L. T. Biegler, A. Brambilla, \& C. Scali, (Eds.), International symposium on advanced control of chemical processes (ADCHEM) (pp. 905-910).

Banga, J. R., Alonso, A. A., \& Singh, R. P. (1997). Stochastic dynamic optimization of batch and semicontinuous bioprocesses. Biotechnology Progress, 13, 326-335.

Banga, J. R., Irizarry, R., \& Seider, W. D. (1998). Stochastic optimization for optimal and model-predictive 
control. Computers and Chemical Engineering, 22(4-5), 603-612.

Banga, J. R., Alonso, A. A., Gallardo, J. M., \& Martin, R. P. (1993). Mathematical modeling and simulation of the thermal processing of anisotropic and non-homogeneous conduction heated canned foods: application to canned tuna. Journal of Food Engineering, 18(4), 369-387.

Banga, J. R., Martin, R. P., Gallardo, J. M., \& Casares, J. J. (1991). Optimization of thermal processing of conduction-heated canned foods: Study of several objective functions. Journal of Food Engineering, $14(1), 25-51$.

Chalabi, Z. S., Willigenburg, L. V., \& Straten, G. V. (1999). Robust optimal receding horizon control of the thermal sterilization of canned foods. Journal of Food Engineering, 40(3), 207-218.

Durance, T. D. (1997). Improving canned food quality with variable retort temperature processes. Trends in Food Science and Technology, 8(4), 113-118.

Hindmarsh, A. (1983). ODEPACK, a systematized collection of ODE solvers (pp. 55-64). Amsterdam: North Holland.

Kleis, D., \& Sachs, E. W. (2000). Optimal control of the sterilization of prepackaged food. SIAM Journal on Optimization, 10(4), 1180-1195.

Nadkarni, M. M., \& Hatton, T. A. (1985). Optimal nutrient retention during the thermal processing of conduction-heated canned food: application of the distributed minimum principle. Journal of Food Science, 50, 1312-1321.

Saguy, I., \& Karel, M. (1979). Optimal retort temperature profile in optimizing thiamine retention in conduction-type heating of canned foods. Journal of Food Science, 44, 1485-1490.

Schiesser, W. (1991). The numerical method of lines. New York: Academic Press.

Silva, C. L. M., Oliveira, F., \& Hendrickx, M. (1993). Modelling optimum processing conditions for the sterilization of prepackaged foods. Food Control, 4(2), 67-78.

Stumbo, C. R. (1973). Thermobacteriology in food processing. New York: Academic Press.

Teixeira, A. A., Zinsmeister, G. E., \& Zahradnik, J. W. (1975). Computer simulation of variable retort control and container geometry as a possible means of improving thiamine retention in thermally processed foods. Journal of Food Science, 40, 656-659.

Teixeira, A. A., Dixon, J. R., Zahradnik, J. W., \& Zinsmeister, G. E. (1969). Computer optimization of nutrient retention in thermal processing of conduction-heated foods. Food Technology, 23, 137-142.

Terajima, Y., \& Nonaka, Y. (1996). Retort temperature profile for optimum quality during conductionheating of foods in retortable pouches. Journal of Food Science, 61(4), 673-678. 
Nomenclature

$\mathrm{a}_{\omega \mathrm{i}}, \mathrm{a}_{\Theta \mathrm{i}}, \mathrm{a}_{\mathrm{Ni}} \quad$ time coefficients in the series approximation of $\omega, \Theta$ and $\ln \mathrm{C}_{\mathrm{N}}$, respectively

$\mathrm{b}_{\omega \mathrm{ij}}, \mathrm{c}_{\omega \mathrm{i}} \quad$ coefficients appearing in the reduced-order model equations for $\omega$

$b_{\Theta i j}, b_{i j k}, c_{\Theta i} \quad$ coefficients appearing in the reduced- order model equations for $\Theta$

$\mathrm{b}_{\mathrm{Nij}}, \mathrm{c}_{\mathrm{Ni}} \quad$ coefficients appearing in the reduced-order model equations for $\ln \mathrm{C}_{\mathrm{N}}$

$\mathrm{C}_{\mathrm{i}} \quad$ microbial spores or nutrient concentration

$\mathrm{C}_{\mathrm{p}} \quad$ specific heat capacity

$\mathrm{D}_{\mathrm{i}, \text { ref }} \quad$ time required to reduce the concentration by a factor of 10 at a certain

temperature

$\mathrm{F}_{\mathrm{c}} \quad$ lethality at the critical point

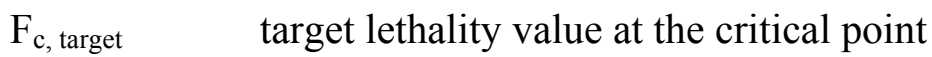

$\mathrm{F}_{\mathrm{S}} \quad$ mass average lethality

$\mathrm{J} \quad$ objective function in the optimization problem

$\mathrm{K}(\mathrm{T}) \quad$ thermal conductivity

L(.) Laplacian operator

$\mathrm{N}_{\omega}, \mathrm{N}_{\Theta}, \mathrm{N}_{\mathrm{N}}$ number of eigenfunctions to approximate $\omega, \Theta$ and $\ln \mathrm{C}_{\mathrm{N}}$, respectively

ret $_{\mathrm{N}} \quad$ average nutrient retention

r; z spatial cylindrical coordinates

$\mathrm{t} \quad$ time

$\mathrm{t}_{\mathrm{f}} \quad$ final time, total process time

$\mathrm{t}_{\mathrm{h}} \quad$ heating time

T temperature

$\mathrm{T}_{\mathrm{c}} \quad$ temperature at the critical point

$\mathrm{T}_{\text {heating heating temperature }}$

$\mathrm{T}_{\mathrm{i}, \mathrm{ref}} \quad$ reference temperature

$\mathrm{T}_{\text {retort }} \quad$ temperature profile inside the retort during a thermal process

$\mathrm{V} \quad$ volume

$\mathrm{Z}_{\mathrm{i}, \text { ref }} \quad$ temperature increase necessary for reducing $\mathrm{D}_{\mathrm{i}, \text { ref }}$ by a factor of 10

Greeks

$\rho \quad$ density

$\theta \quad$ cylindrical coordinate

$\alpha \quad$ thermal diffusivity 
transformed variable: linear function of the temperature transformed variable: exponential function of the temperature

(1)., $\leftrightarrow$. and $\ln C_{N}$ series approximation of $\omega, \Theta$ and $\ln C_{N}$, respectively

$\phi_{\omega \mathrm{i}}, \phi_{\Theta \mathrm{i}}, \phi_{\mathrm{Ni}}$ empirical eigenfunctions for the aproximation of $\omega, \mathrm{H}$ and $\ln \mathrm{C}_{\mathrm{N}}$, respectively

$\partial$ partial derivative

Table 1 Sterilization of pork puree: problem parameters

\begin{tabular}{ll} 
Can radius $(\mathrm{R}, \mathrm{m})$ & 0.04375 \\
Can height $(2 \mathrm{~L}, \mathrm{~m})$ & 0.1160 \\
$\alpha\left(\mathrm{m}^{2} \mathrm{~s}^{-1}\right)$ & $1.5443 \times 10^{7}$ \\
$\mathrm{~T}_{0}\left({ }^{\circ} \mathrm{C}\right)$ & 71.11 \\
Microorganism & Bacillus stearothermophilus \\
$\mathrm{z}_{\mathrm{M}, \text { ref }}\left({ }^{\circ} \mathrm{C}\right)$ & 10.0 \\
$\mathrm{D}_{\mathrm{M}, \text { ref }}(\mathrm{s})$ & 240.0 \\
$\mathrm{~T}_{\mathrm{M}, \text { ref }}\left({ }^{\circ} \mathrm{C}\right)$ & 121.11 \\
$\mathrm{Nutrient}^{\circ}$ & $\mathrm{Thiamine}$ \\
$\mathrm{z}_{\mathrm{N}, \text { ref }}\left({ }^{\circ} \mathrm{C}\right)$ & 25.56 \\
$\mathrm{D}_{\mathrm{N}, \text { ref }}(\mathrm{s})$ & 10716.0 \\
$\mathrm{~T}_{\mathrm{N}, \text { ref }}\left({ }^{\circ} \mathrm{C}\right)$ & 121.11 \\
\hline
\end{tabular}

Fig. 1. Typical snapshots for $\omega$

Fig. 2. Typical snapshots for $\Theta$

Fig. 3. Typical snapshots for $\mathrm{C}_{\mathrm{N}}$

Fig. 4. Relative energy versus number of empirical eigenfunctions for the three variables: $\omega, \Theta$ and $\ln C_{N}$.

Fig. 5. Empirical eigenfunctions for temperature projection.

Fig. 6. Empirical eigenfunctions for $\Theta$ projection 
Fig. 7. Empirical eigenfunctions for $\ln \mathrm{C}_{\mathrm{N}}$ projection

Fig. 8. Typical constant retort temperature profile during a sterilization process.

Fig. 9. Comparison of the reduced and the nominal models: temperature evolution in a number of points inside the food.

Fig. 10. Comparison of the reduced and the nominal models: lethality at the critical point $\left(\mathrm{F}_{\mathrm{c}}(\mathrm{t})\right)$.

Fig. 11. Comparison of the reduced and the nominal models: average nutrient retention $\left(\operatorname{ret}_{\mathrm{N}}(\mathrm{t})\right)$

Fig. 12. CRT processes: nutrient retention versus heating temperature for a number of $\mathrm{F}_{\mathrm{c}}$, target values.

Fig. 13. CRT processes: heating time versus heating temperature for a number of $F_{c}$, target values

Fig. 14. Optimal control (retort temperature) profile and temperature at the critical point, obtained with the reduced-order model.

Fig. 15. Average nutrient retention for the optimal control profile

Fig. 16. Lethality at the critical point for the optimal control profile

Fig. 17. Curves of convergence for the dynamic optimization: reduced model versus nominal model 